\title{
Endothelial cell pyroptosis plays an important role in Kawasaki disease via HMGB1/RAGE/cathespin B signaling pathway and NLRP3 inflammasome activation
}

Chang Jia', Jian Zhang ${ }^{2}$, Huanwen Chen ${ }^{3}$, Yingzhi Zhuge ${ }^{2}$, Huiqiao Chen², Fanyu Qian², Kailiang Zhou', Chao Niu', Fangyan Wang ${ }^{5}$, Huixian Qiu' ${ }^{2}$, Zhenquan Wang ${ }^{2}$, Jian Xiao (1) ${ }^{6}$, Xing Rong ${ }^{2}$ and Maoping Chu $\mathbb{B}^{1,2}$

\begin{abstract}
Kawasaki disease (KD) is the most common cause of pediatric cardiac disease in developed countries, and can lead to permanent coronary artery damage and long term sequelae such as coronary artery aneurysms. Given the prevalence and severity of KD, further research is warranted on its pathophysiology. It is known that endothelial cell damage and inflammation are two essential processes resulting in the coronary endothelial dysfunction in KD. However, detailed mechanisms are largely unknown. In this study, we investigated the role of pyroptosis in the setting of KD, and hypothesized that pyroptosis may play a central role in its pathophysiology. In vivo experiments of patients with KD demonstrated that serum levels of pyroptosis-related proteins, including ASC, caspase-1, IL-1 $\beta$, IL-18, GSDMD and lactic dehydrogenase (LDH), were significantly increased in $\mathrm{KD}$ compared with healthy controls (HCs). Moreover, western blot analysis showed that the expression of GSDMD and mature IL-1 $\beta$ was notably elevated in KD sera. In vitro, exposure of human umbilical vein endothelial cells (HUVECs) to KD sera-treated THP1 cells resulted in the activation of NLRP3 inflammasome and subsequent pyroptosis induction, as evidenced by elevated expression of caspase-1, GSDMD, cleaved p30 form of GSDMD, IL-1 $\beta$ and IL-18, and increased LDH release and TUNEL and propidium iodide (PI)-positive cells. Furthermore, our results showed that NLRP3-dependent endothelial cell pyroptosis was activated by HMGB1/RAGE/cathepsin B signaling. These findings were also recapitulated in a mouse model of KD induced by Candida albicans cell wall extracts (CAWS). Together, our findings suggest that endothelial cell pyroptosis may play a significant role in coronary endothelial damage in KD, providing novel evidence that further elucidates its

pathophysiology.
\end{abstract}

Correspondence: Xing Rong (rstar1978mail@163.com) or

Maoping Chu (chmping@hotmail.com)

${ }^{1}$ Pediatric Research Institute, The Second Affiliated Hospital and Yuying

Children's Hospital of Wenzhou Medical University, Wenzhou 325027, China

${ }^{2}$ Children's Heart Center, Institute of Cardiovascular Development and

Translational Medicine, The Second Affiliated Hospital and Yuying Children's Hospital of Wenzhou Medical University, Wenzhou 325027, China

Full list of author information is available at the end of the article

These authors contributed equally: Chang Jia, Jian Zhang

Edited by A. Stephanou

\section{Introduction}

Kawasaki disease (KD) is an acute vasculitis and is the main cause of acquired heart disease in pediatric populations of developed countries ${ }^{1}$. Coronary artery lesions are the most severe complications in children with $\mathrm{KD}$, and non-specific immune-suppressive therapy with intravenous immunoglobulin (IVIG) remains the mainstay treatment. Without early intervention, $25 \%$ of $\mathrm{KD}$ patients would go on to develop coronary artery aneur$\mathrm{ysm}^{2}$, and even with prompt administration of IVIG, risk of coronary artery aneurysm remains unacceptable high

\section{(-) The Author(s) 2019}

(c) Open Access This article is licensed under a Creative Commons Attribution 4.0 International License, which permits use, sharing, adaptation, distribution and reproduction cc) in any medium or format, as long as you give appropriate credit to the original author(s) and the source, provide a link to the Creative Commons license, and indicate if changes were made. The images or other third party material in this article are included in the article's Creative Commons license, unless indicated otherwise in a credit line to the material. If material is not included in the article's Creative Commons license and your intended use is not permitted by statutory regulation or exceeds the permitted use, you will need to obtain permission directly from the copyright holder. To view a copy of this license, visit http://creativecommons.org/licenses/by/4.0/. 
$(4 \%)^{3}$. Part of the difficulty of treating KD is due to its complex inflammatory pathophysiology. Previous studies reported that endothelial cell injury and inflammation are the two key pathological mechanisms for $\mathrm{KD}^{4,5}$. While some mechanisms for endothelial cell injury in KD, such as endothelial-mesenchymal transition (EndoMT) ${ }^{2}$ and endothelial cell apoptosis ${ }^{6,7}$, have been demonstrated, whether other mechanisms also play a role in this complex inflammatory disease remains unknown.

$\mathrm{KD}$, an inflammatory disease, involves the activation of innate and adaptive immunity, which eventually causes the release of cytokine factors and establishes an inflammatory environment. Among them, the cytokine factors released by monocyte/macrophages play an important role in vascular endothelial damage during acute $\mathrm{KD}^{8,9}$, and THP1 cell line has been widely used as an in vitro model of human monocytes and macrophage in mechanistic studies of inflammatory disease ${ }^{10}$. As is known, acute inflammation in $\mathrm{KD}$ is accompanied by marked endothelial cell dysfunction and production of cytokines, such as interleukin (IL)-1 $\beta^{11,12}$, which may suggest involvement of pyroptosis in the pathophysiology of KD. Pyroptosis is a form of proinflammatory cell death that combines features of both apoptosis and necrosis, and can be mediated by inflammasome-dependent caspase- 1 activation ${ }^{13}$, which is responsible for the cleavage and/or maturation of Gasdermin D (GSDMD), IL-1 $\beta$ and IL-18. Pyroptosis has been shown to play important roles in cardiovascular diseases, such as atherosclerosis ${ }^{14,15}$, diabetic cardiomyopathy ${ }^{16}$, and myocardial ischemia/reperfusion injury ${ }^{17}$. However, it remains unclear whether the coronary endothelial injury seen in KD is associated with pyroptosis.

Canonical pyroptosis, which is caspase- 1 mediated, can be activated by seven kinds of inflammasomes, including NLRP3, NLRP1, NLRP6, NLRP9, AIM2, NLRC4, and Pyrin $^{13}$. Among them, NLRP3 is identified as an important NOD-like receptor protein that can recognize both microbial and non-microbial danger signals and trigger a sterile inflammatory response. In a Lactobacillus casei ( $L$. casei) cell wall extract (LCWE)-induced KD mouse model, NLRP3 inflammasome is activated in the coronary arterial endothelium and leads to a significant increase in caspase1 activity and IL-1 $\beta$ production. This phenotype is rescued by Nlrp3 gene silencing, lysosome membrane stabilizing agents, and cathepsin B silencing, indicating that LCWE induces lysosomal membrane permeabilization and subsequent lysosomal cathepsin B release, eventually leading to the activation of NLRP3 inflammasome, which then contribute to coronary arteritis in $\mathrm{KD}^{18}$. Pyroptosis is also known as gasdermin-mediated programmed necro$\mathrm{sis}^{19}$, and whether the activation of NLRP3 inflammasome also leads to endothelial necrosis is unclear in KD. Furthermore, the mechanism of lysosomal membrane rupture and cathepsin B release is also undefined.
High mobility group box 1 (HMGB1) is a highly conserved $30 \mathrm{kDa}$ nuclear protein that can act as an extracellular signal after being released by immune cells or necrotic cells ${ }^{20}$. The released HMGB1 can mediate the proinflammatory responses of endothelial cells ${ }^{21}$, and induce apoptotic, autophagic or pyroptotic cell death ${ }^{22-24}$. In addition, HMGB1 can trigger the activation of NLRP3 and AIM2 inflammasomes after binding various DNA sequences in a TLR9 or RAGE-dependent manner ${ }^{25}$. Xu et al. reported that HMGB1-induced macrophage pyroptosis acts through RAGE/dynamin/cathepsin B signaling, which then triggers pyroptosome formation and caspase-1-mediated pyroptosis ${ }^{21}$. It has been reported that the serum contents of HMGB1 in KD patients are increased in early acute phase and declined after defervescence. Moreover, the mRNA expression of its receptor, RAGE, is remarkably upregulated in KD patients ${ }^{26}$. Therefore, we speculate that endothelial cell pyroptosis is induced in a NLRP3-dependent manner in KD, which is mediated by HMGB1/RAGE/cathepsin B signaling pathway.

To test our hypothesis, we first examined the levels of pyroptosis-related proteins, including ASC, caspase-1, IL$1 \beta$, IL-18, GSDMD, and lactic dehydrogenase (LDH), in the sera of KD patients as compared with healthy controls $(\mathrm{HCs})$, and analyzed the expression of GSDMD and mature IL-1 $\beta$ by western blot analysis. Then, we confirmed pyroptosis induction in endothelial cells treated with KD-like conditions, and observed the pyroptotic changes after inhibiting NLRP3, cathepsin B, RAGE or HMGB1. To further verify the role of endothelial cell pyroptosis in $\mathrm{KD}$, we detected the phenotypes and mechanisms in a murine model of KD induced by Candida albicans cell wall extracts (CAWS).

\section{Results}

Serum levels of pyroptosis-related proteins are increased in $\mathrm{KD}$

During pyroptosis, the levels of ASC, activated caspase1 , cleaved IL-1 $\beta$, IL-18, and gasdermin D (GSDMD) are expected to be significantly upregulated ${ }^{14}$. To preliminarily determine whether pyroptosis occurs in $\mathrm{KD}$, the serum levels of circulating pyroptosis-related proteins were measured and compared between KD patients and age-matched healthy controls (HCs). Results showed that the levels of total ASC, caspase-1, IL-1 $\beta$, IL-18, and GSDMD were remarkably increased in sera from KD subjects compared with HC (Fig. 1a-e). Since damaged cell membrane in pyroptotic cells can lead to the release of intracellular contents ${ }^{27}$, including lactic dehydrogenase (LDH), LDH release assay was conducted. Here, we found that LDH levels were also significantly increased in the sera from KD patients (Fig. 1f). Western blot analysis showed that the expression of GSDMD, a key executor of 


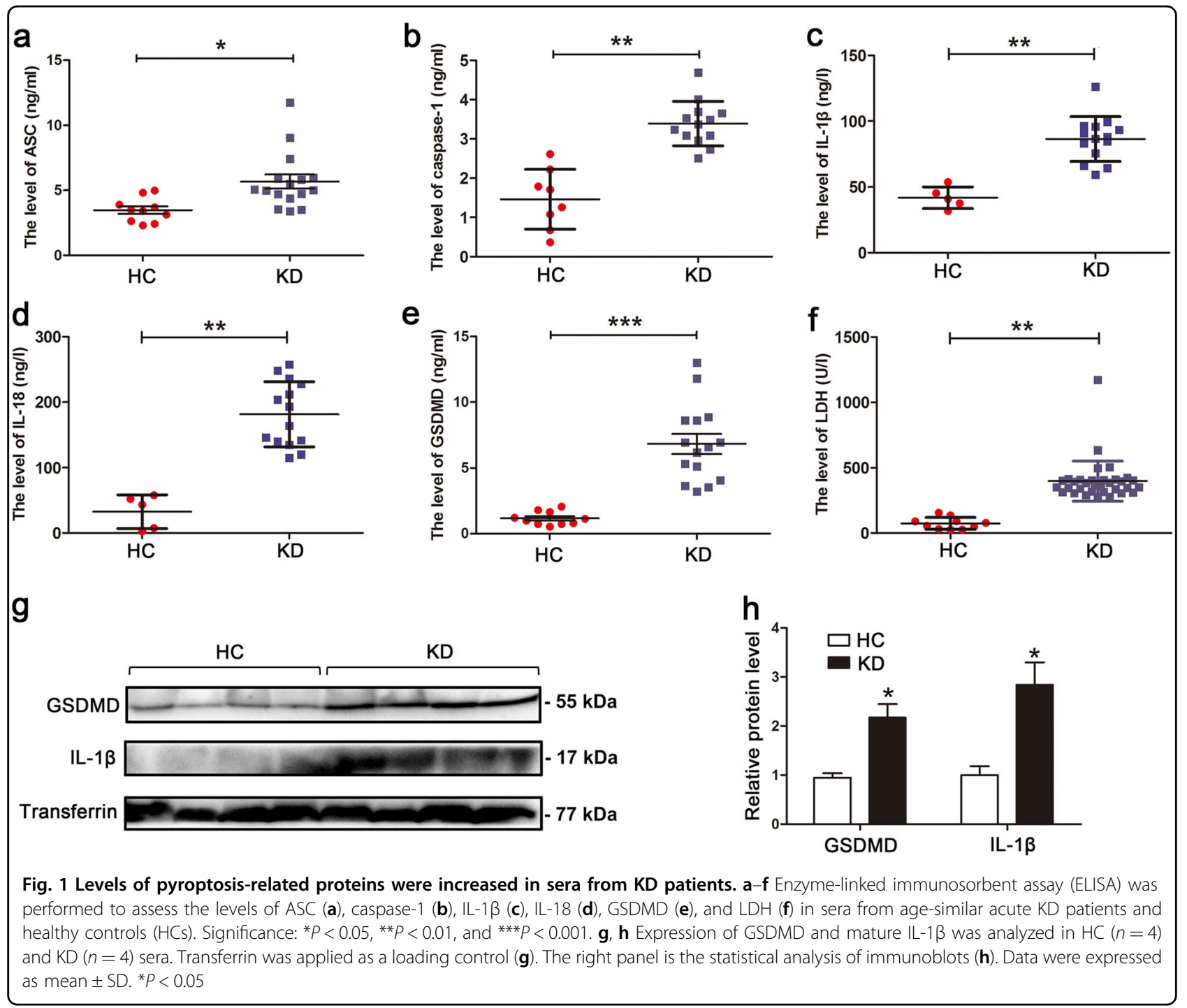

pyroptosis, and mature IL- $1 \beta$ were also remarkably elevated in KD sera (Fig. 1g, h). These results suggested that pyroptosis may be involved in KD.

\section{KD sera-treated THP1 cells induce pyroptosis of endothelial cells}

Next, we conducted in vitro experiments to further investigate the role of pyroptosis in KD. In KD, endothelial cells are exposed to inflammatory conditions. Thus, to re-create the inflammatory environment surrounding endothelial cells in KD, we utilized KD sera-treated THP1 cells and co-cultivated them with human umbilical vein endothelial cells (HUVECs) to create an in vitro coculture experimental system. More specifically, these two cell types were separated by a transwell chamber, with the HUVECs were cultured in the lower chamber, and the THP1 cells were placed in the upper chamber that permits diffusion of soluble molecules. To characterize whether pyroptosis was induced in the endothelial cells (ECs) after co-culturing them with $\mathrm{KD}$ sera-treated THP1 cells (referred to as "KD-treated ECs" from now on), the expression of pyroptosis-related proteins was assessed by western blot analysis. As shown in Fig. 2a, b, the expression levels of NLRP3, caspase-1, GSDMD, cleaved p30 form of GSDMD, IL-1 $\beta$ and IL-18 were notably increased in KD-treated ECs. To further dissect EC pyroptosis, the immunofluorescence staining of caspase- 1 and TUNEL, LDH release and PI staining were respectively evaluated ${ }^{11}$. Results showed that caspase- 1 fluorescence intensity, TUNEL-positive cells, release of $\mathrm{LDH}$, and PI-positive cells were remarkably elevated by KD conditions (Fig. 2c-f). To further substantiate that pyroptosis was implicated in the above phenotypes, GSDMD-derived pyroptosis inhibitors, necrosulfonamide and Ac-FLTD-CMK, were utilized to observe EC viability. As shown in Figs. S1 and S2, the addition of these 

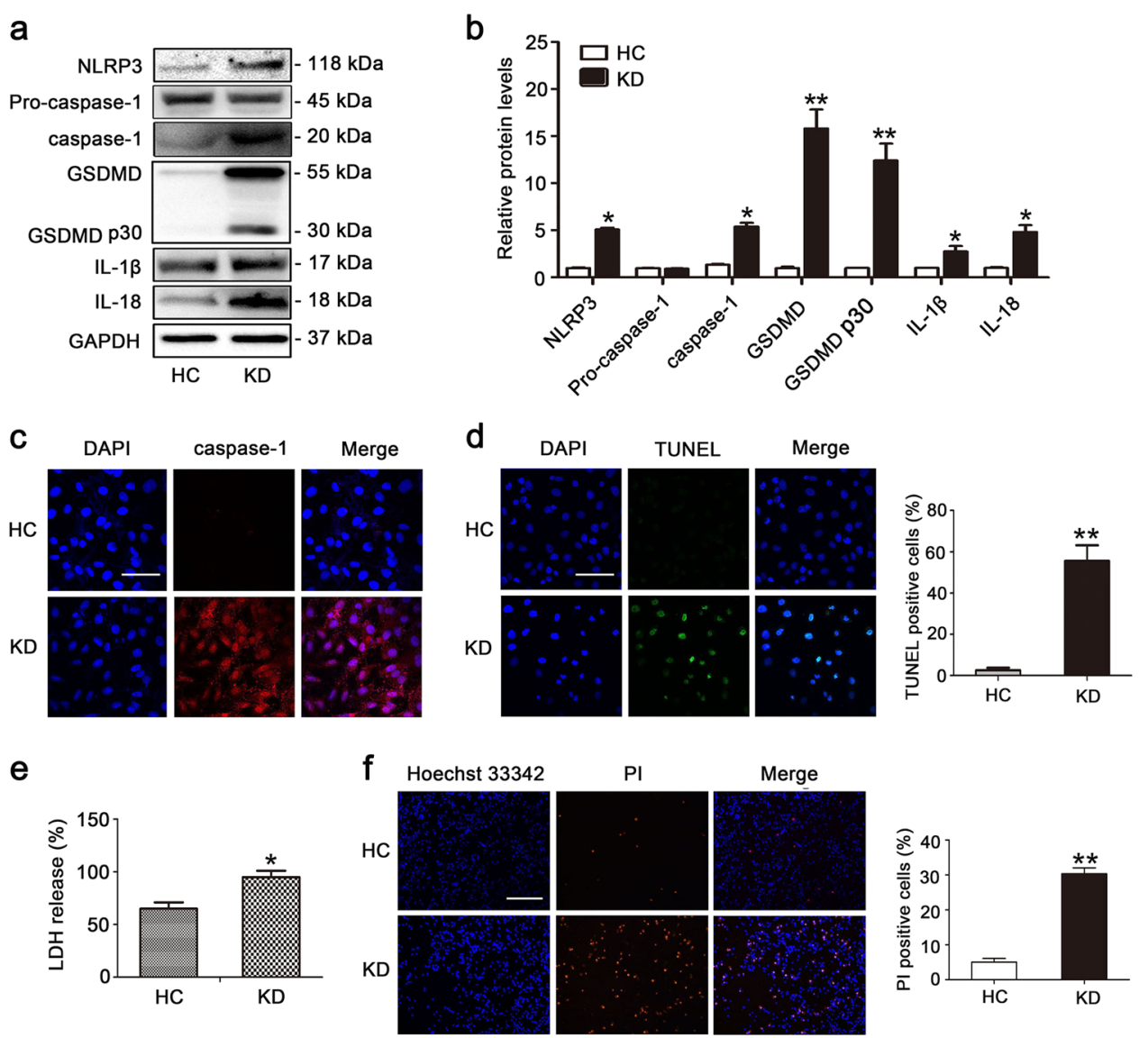

Fig. 2 Pyroptosis occurred in human umbilical vein endothelial cells (HUVECs) after exposure to KD sera-treated THP1 cells. a, b HUVECS were co-cultured with THP1 cells that were treated with HC or KD sera (pooled from eight patients) for $24 \mathrm{~h}$. Protein levels of NLRP3, caspase-1, mature IL-1 $\beta$, and IL-18, and cleavage of GSDMD were examined by western blot analysis. GAPDH was used as an internal control. Data in (b) were shown as mean \pm SD from three independent experiments involving different batches of cells but the same pooled $\mathrm{HC}$ or $\mathrm{KD}$ sera. ${ }^{*} P<0.05$ and ${ }^{*} P<0.01$. c $\mathbf{d}$ Caspase-1 fluorescence intensity and the percentage of TUNEL-positive cells were respectively evaluated using immunofluorescent assay and TUNEL staining. The nuclei were stained blue using DAPI. Magnification: $\times 200$. Scale bar $=100 \mu \mathrm{m}$. ${ }^{*} P<0.01$. e LDH release was determined in HUVECs after HC or KD sera treatment $(n=5)$. ${ }^{P}<0.05$. f Percentage of PI-positive cells was detected in HUVECs using Hoechst33342/PI staining. Magnification: $\times 100$. Scale bar $=500 \mu \mathrm{m}$. Significance: ${ }^{* *} P<0.01$

pyroptosis inhibitors significantly decreased LDH release, increased cell viability, alleviated cell death and declined IL-1 $\beta$ release. Together, these results suggested that pyroptosis-related cell death occurred in KD-treated ECs in our in vitro model of KD.

\section{NLRP3 inflammasome activation triggers downstream activation of pyroptosis in KD-treated ECs}

NLRP3 inflammasome is known to recruit and activate caspase- 1 through ASC, and activated caspase- 1 can then cleave GSDMD, IL-1 $\beta$, and IL-18 to their mature forms and subsequently trigger pyroptotic cell death ${ }^{28}$. Given our results confirming the concurrent activation of NLRP3, caspase-1, IL-1 $\beta$, IL-18, and GSDMD, we hypothesized that NLRP3 inflammasome may be triggered upstream of pyroptosis activation. To substantiate whether caspase-1 activation and subsequent pyroptosis were mediated by upstream NLRP3 inflammasome activation, we performed NLRP3 inhibitory experiments with the NLRP3 inhibitor MCC950. Results showed that MCC950 addition notably decreased the protein level of NLRP3, activated caspase-1, GSDMD, and cleaved p30 form of GSDMD, and inhibited the maturation of IL- $1 \beta$ and IL-18 (Fig. 3a-h). Moreover, caspase-1 fluorescence intensity and the percentage of TUNEL-positive cells were obviously reduced by treatment with MCC950 in KDtreated ECs (Fig. 3i, j). What's more, LDH release and PIpositive cells were reduced as well (Fig. $3 \mathrm{k}, \mathrm{l}$ ), indicating that the cell membrane rupture and cell lysis were reversed by MCC950. Together, these data provided strong evidence that EC pyroptosis in KD conditions was downstream effect of NLRP3 inflammasome activation. 


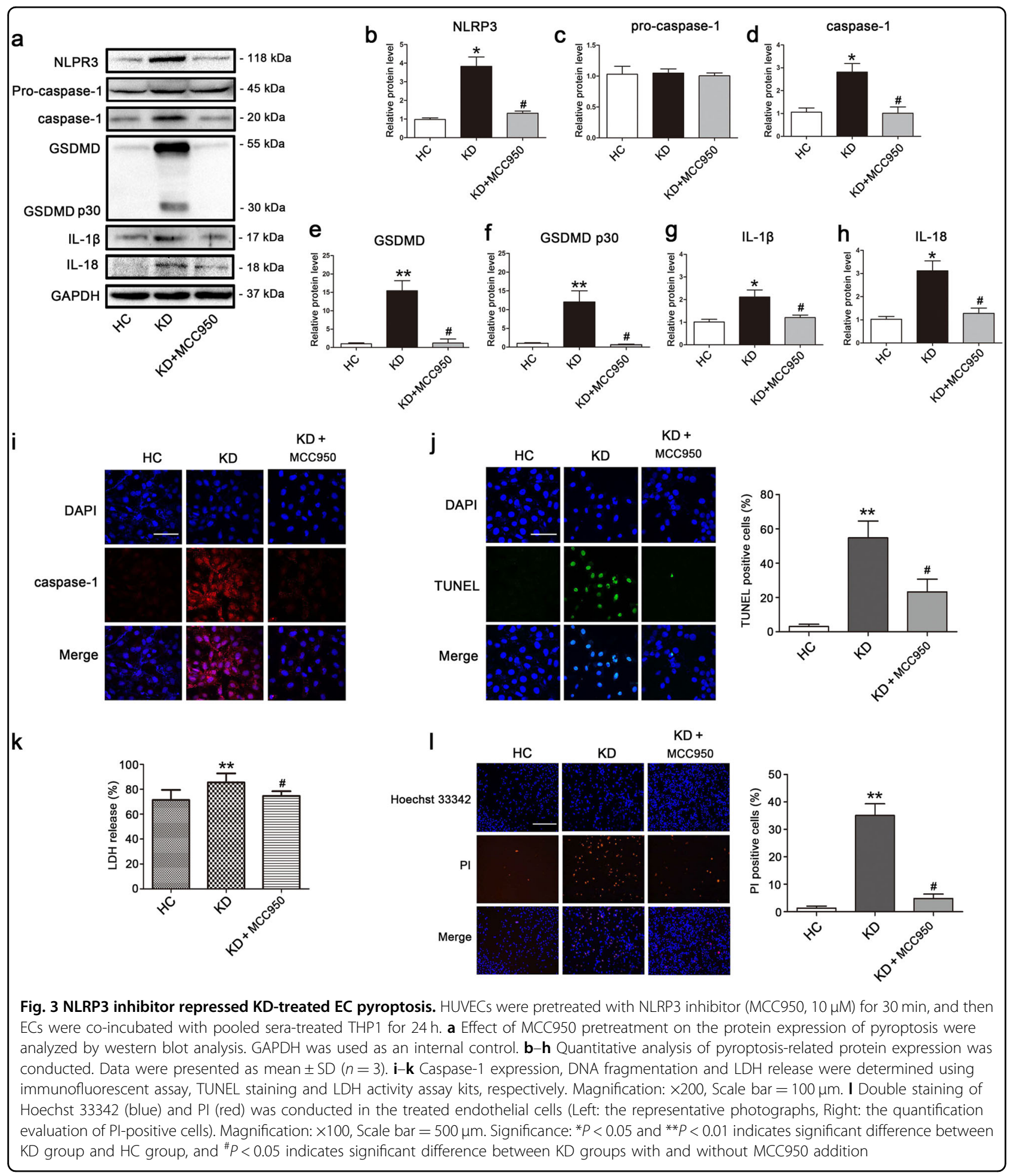

Pyroptosis of KD-treated ECs is dependent on cathepsin B activation

Lysosome rupture, and cathepsin B activation and release from lysosomes are important mechanisms for the activation of NLRP3 inflammsome ${ }^{29}$. Previous study has reported that endothelial NLRP3 inflammasome activation is related to lysosomal destabilization and consequent release of lysosomal cathepsin B in an animal model of KD (coronary arteritis induced by LCWE) ${ }^{18}$. To address whether NLRP3 inflammasome-mediated EC pyroptosis 
found in our model is associated with cathepsin B activation in $K D$, cathepsin $B$ activity in the cytoplasm was examined using Magic Red Cathepsin B detection reagent. KD-treated ECs exhibited an obvious cytosolic pattern of activated cathepsin B staining compared with cells treated with control while the addition of cathepsin B specific inhibitor CA074-Me significantly inhibited cathepsin B activity (Fig. 4a). Moreover, when ECs were pretreated with CA074-Me, the protein levels of pyroptosis-related proteins, including NLRP3, caspase-1, GSDMD, cleaved p30 form of GSDMD, mature IL-1 $\beta$ and IL-18, were remarkably decreased in KD sera-treated cells (Fig. 4b-i). Furthermore, caspase- 1 fluorescence intensity and DNA fragmentation detected by TUNEL were significantly attenuated after pretreatment with CA074-Me (Fig. 4j, k). In addition, the level of LDH release and cell membrane damage indicated by PI staining were also decreased by CA074-Me (Fig. 4l, m). These results confirmed a key role of cathepsin $\mathrm{B}$ for regulating pyroptosis in $\mathrm{KD}$.

\section{Pyroptosis of KD-treated ECs is mediated by HMGB1/RAGE signaling}

It is well known that serum levels of HMGB1 are increased in KD during the early acute phase and gradually declines after defervescence. In particular, the expression of one of HMGB1 receptors, RAGE, is upregulated in KD patients ${ }^{26}$, suggesting that HMGB1-RAGE signaling may play an important role in the pathophysiology of KD. Previous studies demonstrated that HMGB1-induced macrophage pyroptosis is mediated by RAGE-dependent signaling, which can initiate HMGB1 endocytosis and subsequent cathepsin B release from damaged lysosomes ${ }^{24}$. To decipher whether our results of pyroptosis induction in KD-treated ECs is related to HMGB1-RAGE signaling, HMGB1 levels in the supernatant medium of KD sera-treated THP1 cells, and RAGE expression in ECs were determined. Results showed that HMGB1 contents were significantly increased in the supernatant of THP1 cells after treatment with KD sera (Fig. 5a), and the mRNA level and protein expression of RAGE was notably elevated in ECs after treatment with KD conditions (Fig. 5b, c). To determine whether HMGB1/RAGE signaling is responsible for pyroptosis in KD-treated ECs, we pretreated ECs with a RAGE-specific inhibitor (FPS-ZM1) or an anti-HMGB1 neutralizing antibody. Then, we measured cathepsin B activity, along with the expression levels of pyroptosis-related proteins, including NLRP3, caspase-1, GSDMD, cleaved p30 form of GSDMD, mature IL-1 $\beta$ and IL-18. Results showed that these parameters were remarkably reduced in KD-treated ECs (Fig. 5d-1). Moreover, the activated caspase-1 fluorescence intensity, DNA fragmentation, LDH release, and membrane rupture were also remarkably alleviated after inhibition of RAGE by FPS-ZM1 or neutralization of
HMGB1 by the anti-HMGB1 antibody (Fig. 5m-p). Together, these results demonstrated EC pyroptosis in KD was realized by HMGB1/RAGE signaling, which resulted in subsequent cathepsin B activation and NLRP3mediated EC pyroptosis.

\section{EC pyroptosis is NLRP3-dependent in a KD mouse model}

To further explore the role of pyroptosis in a mouse model of KD, Candida albicans cell wall extracts (CAWS) were used to induce coronary arteritis as described before $^{5}$. Consistent with previous studies, CAWS treatment obviously induced coronary arteritis. However, the addition of NLRP3 inhibitor MCC950 significantly attenuated coronary artery inflammation (Fig. 6a). Further study found the involved inflammatory cells included macrophages (Fig. 6b) and neutrophils (Fig. 6c). To investigate whether the mitigated coronary arteritis was associated with pyroptosis, the expressions of pyroptosisrelated proteins were determined. As anticipated, the protein levels of NLRP3, activated caspase-1, GSDMD, cleaved p30 form of GSDMD, IL-1 $\beta$, and IL-18 were significantly upregulated in the KD mouse model, and MCC950 treatment significantly decreased the expression of these proteins (Fig. 6d-k). To further demonstrate that pyroptosis occurred in coronary endothelium, CD31/ caspase-1 double staining and CD31/TUNEL double staining were performed. As shown in Fig. 61, m, the expression level of activated caspase- 1 and the percentage of TUNEL-positive cells were markedly increased in the coronary endothelium of the KD mouse model, and the supplementation of MCC950 significantly decreased active caspase-1 level and TUNEL-positive cells. Collectively, these results indicated that coronary EC pyroptosis played a role in the KD mouse model, and that pyroptotic cell death in ECs was mediated by the NLRP3 inflammasome.

\section{NLRP3 inflammasome-mediated EC pyroptosis is realized through HMGB1/RAGE/cathepsin B axis in the KD mouse model}

To confirm whether our in vitro observation of HMGB1/RAGE/cathepsin B signaling-mediated EC pyroptosis also occurs in vivo, KD mice were intraperitoneally injected with a cathepsin B inhibitor (CA074$\mathrm{Me}$ ), a RAGE-specific inhibitor (FPS-ZM1), or the neutralizing antibody against HMGB1 60 min before CAWS treatment. Results showed that the addition of these inhibitors significantly alleviated coronary inflammation (Fig. 7a), and the adhesion of macrophages and neutrophils (Fig. 7b, c). Moreover, the expression of pyroptosis-related proteins was also obviously downregulated after treatment with these inhibitors in the $\mathrm{KD}$ mouse model (Fig. 7d-k). Furthermore, pyroptotic cell death in the coronary endothelium was remarkably 


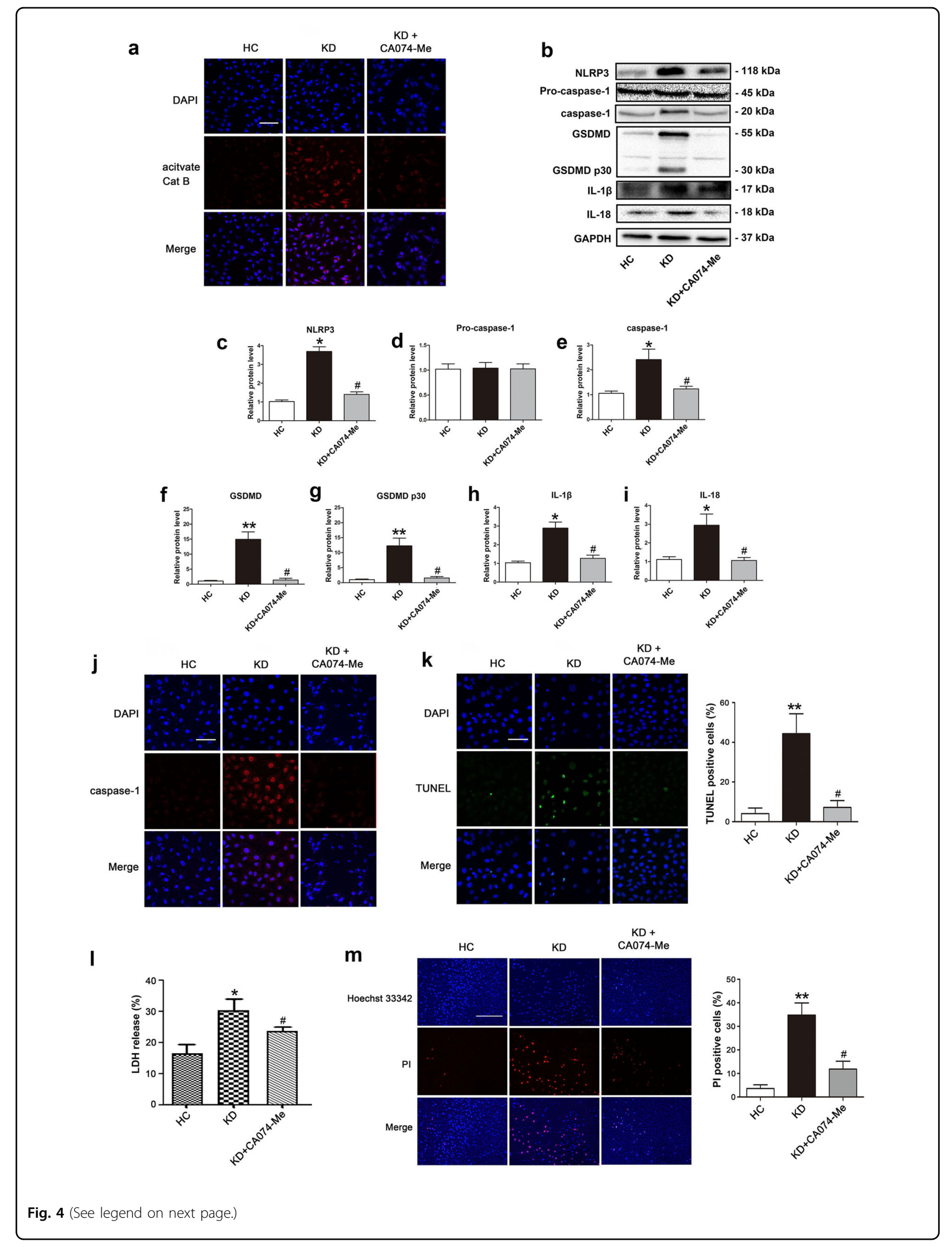


(see figure on previous page)

Fig. 4 Inhibition of cathepsin B alleviated KD-treated EC pyroptosis. HUVECs were pretreated with a cathepsin B inhibitor (CA074-Me, 25 $\mu M$ ) for $30 \mathrm{~min}$, and then ECs were co-cultured with pooled sera-treated THP1 for $24 \mathrm{~h}$. a Endothelial cells were stained with Magic Red Cathepsin B detection reagent to examine the activation of cathepsin B. Magnification: $\times 200$, Scale bar $=100 \mu \mathrm{m}$. $\mathbf{b}$ Effects of CA074-Me treatment on the expression of pyroptosis-associated proteins were assessed in the treated endothelial cells. c-i Quantitative analysis of pyroptosis-related protein expression, and GAPDH was used as an internal control. Data were exhibited as mean \pm SD $(n=3)$. $\mathbf{j}-\mathbf{m}$ Caspase-1 expression, TUNEL staining, LDH release, and Hoechst 33342/PI double staining were conducted as described in Fig. 2c-f. Magnification: $\times 200$, Scale bar $=100 \mu \mathrm{m}$ for (j) and (k). Magnification: $\times 100$, Scale bar $=500 \mu \mathrm{m}$ for $(\mathbf{m})$. Significance: ${ }^{*} P<0.05$, and ${ }^{* *} P<0.01$ indicate significant difference between $\mathrm{KD}$ group and $\mathrm{HC}$ group. ${ }^{~} P<0.05$ indicates significant difference between $\mathrm{KD}$ groups with and without CA074-Me addition

weakened as evidenced by the decreased fluorescence intensity of activated caspase- 1 and reduced percentage of TUNEL-positive cells (Fig. 7l, m). Taken together, these results provided firm evidence that HMGB1/RAGE/ cathepsin B signaling played an important role in activating EC pyroptosis in $\mathrm{KD}$.

\section{Discussion}

In the present study, we demonstrated that EC pyroptosis is a crucial pathophysiological event in $\mathrm{KD}$, and that activation of pyroptosis is triggered by high levels of HMGB1, leading to elevated expression of RAGE and cathepsin B activity, which results in NLRP3 inflammasome-dependent caspase-1-mediated pyroptotic cell death in the ECs (Fig. 8). This previously unappreciated cellular mechanism sheds new light on the pathophysiology of $\mathrm{KD}$, and may open new avenues for the development of techniques to diagnose, evaluate, and treat this potentially devastating disease.

$\mathrm{KD}$ is an acute systemic vasculitis that involves inflammatory environment and endothelial damage. Innate and adaptive immune cells are activated, and inflammatory cytokines are released by a variety of cells, including ECs. These events can be attributed to activation of pyroptosis, and our results showed that pyroptosisrelated parameters were significantly upregulated in the sera from KD patients. To prove upregulation of pyroptosis-related proteins was associated with vascular endothelial damage, monocyte/macrophages (THP1 cell lines) that are essential for the induction of an inflammatory condition in $\mathrm{KD}$ were utilized to simulate the effects of in vivo inflammatory environment on ECs. Results showed that the expression of pyroptosis-related proteins were upregulated in our in vitro model of $\mathrm{KD}$ treated ECs. Attention should be paid to the upregulation of GSDMD in addition to its cleaved form. Previous studies reported that the transcriptional expression of GSDMD is under the control of nuclear factor $\mathrm{kB}$ (NF$\kappa B)^{30}$. The elevated expression of GSDMD revealed that activation of NF- $\mathrm{kB}$ signal in $\mathrm{KD}$, in line with previous reports $^{31}$. To further demonstrate that EC death was associated with pyroptosis, GSDMD-derived pyroptosis inhibitors were added. Addition of these pyroptosis notably inhibited EC death, the expression of pyroptosisrelated parameters, and IL-1 $\beta$ release (Figs. S1 and S2), indicating that pyroptosis occurred in KD-treated ECs. Pyroptotic cell death may be triggered by the activation of canonical caspase-1 and non-canonical caspase-4/511 (human caspase-4/5 and murine caspase-11). In brief, the canonical signaling pathway involves activation of NLRP1, NLRP6, NLRP9, AIM2, NLRC4, Pyrin, and NLRP3. These molecules respond to a variety of signals, such as toxins ${ }^{32}$, microbes $^{33,34}$, double strand RNA and DNA sequen$\mathrm{ces}^{35,36}$, RhoA GTPase ${ }^{37}$, and a variety of other agents. Our data showed that the expression levels of NLRP3 were increased in KD, and inhibition of NLRP3 remarkably decreased the level of pyroptosis-related parameters, and alleviated inflammation in the coronary arteries. These results suggest that EC pyroptosis seen in KD is most likely associated with the activation of NLRP3 inflammasome.

NLRP3 can be activated by crystal phagocytosis, ROS (reactive oxygen species) production, potassium or calcium ion movement, or lysosome disruption and cathepsin $B$ release ${ }^{38}$. Previous studies demonstrated that the NLRP3 activation seen in LCWE-induced KD animal models cannot be ameliorated by potassium channel blockade or ROS scavengers. However, lysosome membrane stabilizing agents and cathepsin B inhibitors can abolish the induced activation of NLRP3 inflammasome in the coronary artery endothelium, indicating NLRP3 activation was mediated by lysosome membrane rupture and the released cathepsin $\mathrm{B}$ in $\mathrm{KD}^{18}$. Activated cathepsin $B$ can regulate the activation of NLRP3 inflammasome directly through interaction with the LRR domain of NLRP $^{29,39}$, or indirectly via calcium signaling ${ }^{40}$ or oxidative stress ${ }^{41}$. Our study showed that the activity of cathepsin B was increased in KD-treated ECs, and the addition of cathepsin B inhibitor significantly downregulate the expression of pyroptosis-related proteins, including NLRP3, activated caspase-1, GSDMD, cleaved p30 form of GSDMD, IL-1 $\beta$, and IL-18. In addition, inhibition of cathepsin B (CA074-Me) also alleviated EC membrane rupture, and coronary artery inflammation was also rescued in our animal model of $\mathrm{KD}$, together suggesting $\mathrm{EC}$ pyroptosis in $\mathrm{KD}$ is mediated by cathepsin $\mathrm{B}$ 


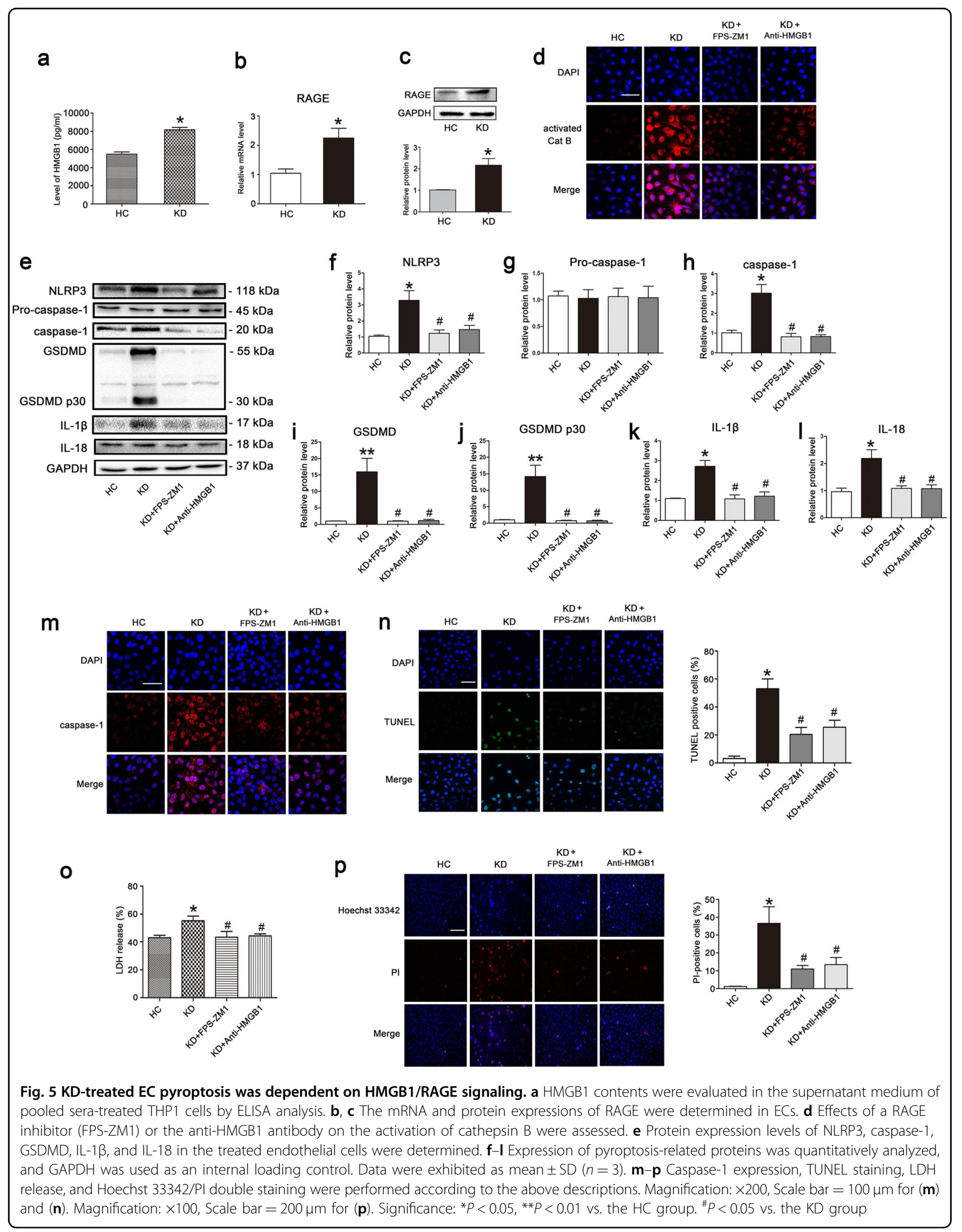




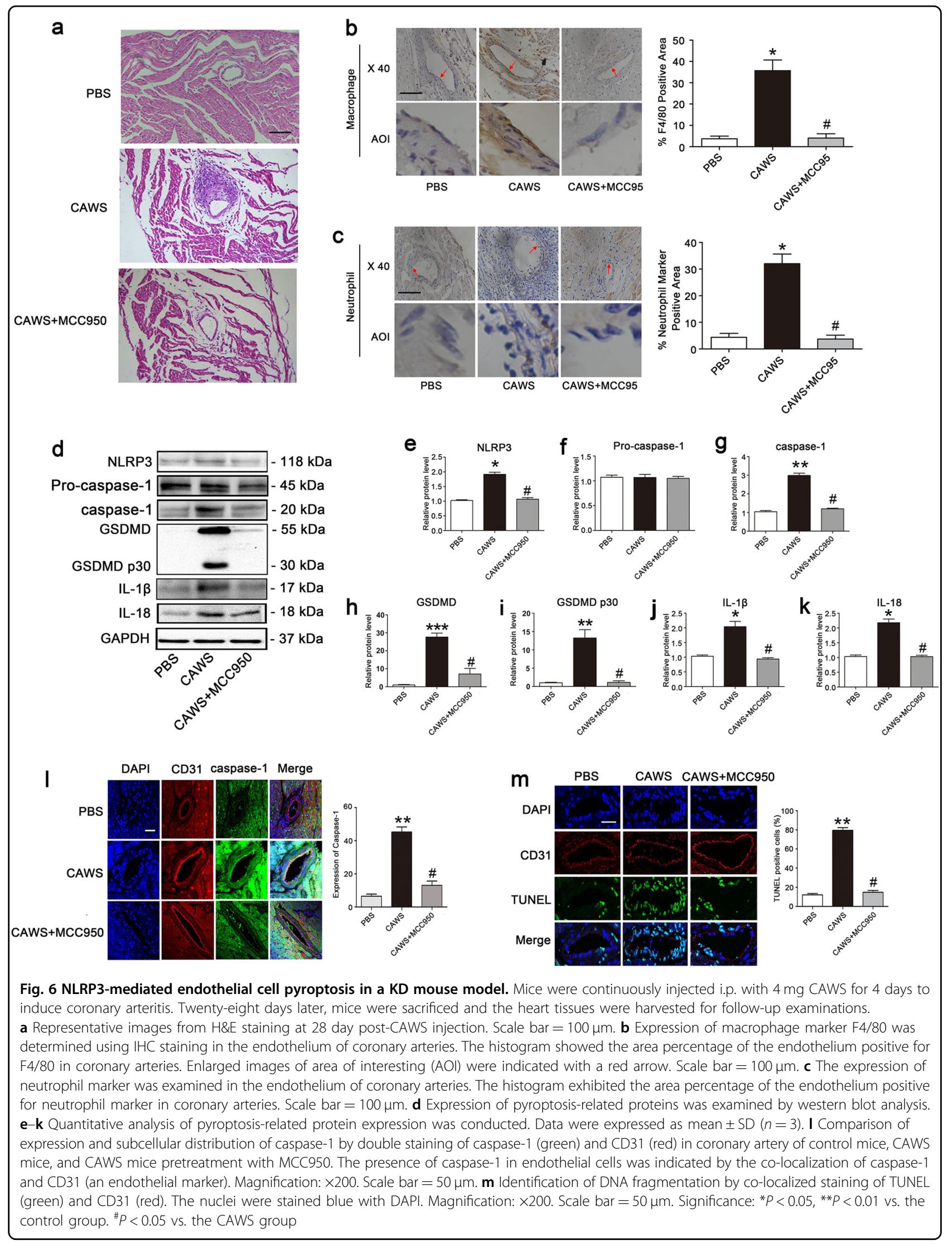




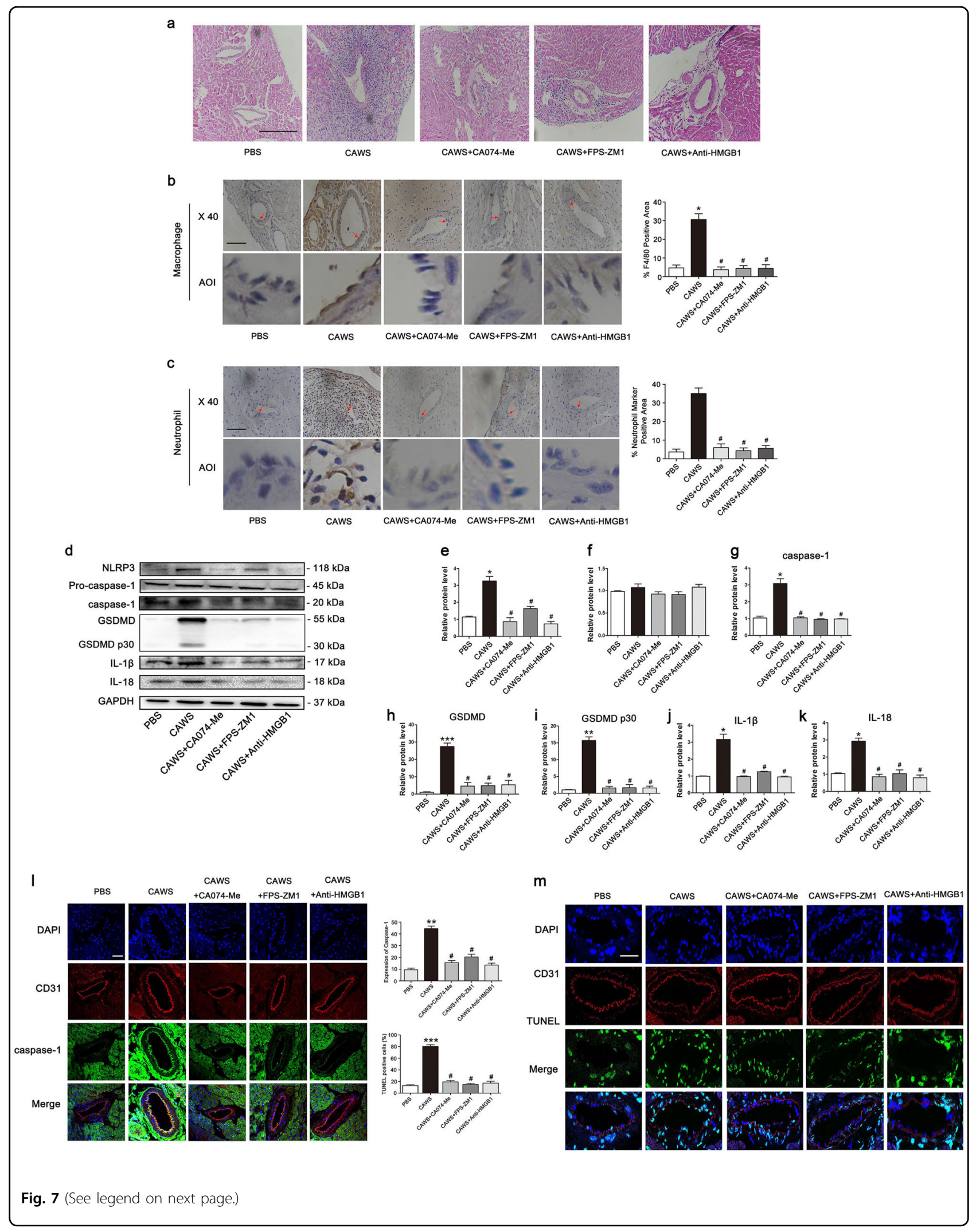


(see figure on previous page)

Fig. 7 HMGB1/RAGE/cathepsin B signaling was implicated in the endothelial cell pyroptosis in the KD mouse model. Mice were pretreated with a cathepsin B inhibitor (CA074-Me), a RAGE-specific inhibitor (FPS-ZM1), and the anti-HMGB1 antibody 60 min before CAWS injection. a Heart tissues were analyzed for inflammatory infiltration by H\&E staining. Magnification: $\times 200$. Scale bar $=200 \mu \mathrm{m}$. $\mathbf{b}$ Expression of macrophage marker F4/ 80 was determined using $\mathrm{IHC}$ staining in the endothelium of coronary arteries. The histogram showed the area percentage of the endothelium positive for F4/80 in coronary arteries. Enlarged images of area of interesting (AOI) were indicated with a red arrow. Scale bar $=100 \mu \mathrm{m}$. c The expression of neutrophil marker was examined in the endothelium of coronary arteries. The histogram exhibited the area percentage of the endothelium positive for neutrophil marker in coronary arteries. Scale bar $=100 \mu \mathrm{m}$. $\mathbf{d}$ Western blot analysis was used to determine the expression of pyroptosis-related proteins. e-k Quantitative analysis was performed to detect pyroptosis-related protein expression. Data were shown as mean \pm SD $(n=3)$. I Caspase-1 expression was analyzed in coronary endothelial cells using caspase-1/CD31 double staining. Magnification: $\times 200$. Scale bar $=$ $50 \mu \mathrm{m}$. $\mathbf{m}$ DNA fragmentation in endothelial cells was identified by co-localization observation of TUNEL and CD31. The nuclei were stained blue with DAPI. Magnification: $\times 200$. Scale bar $=50 \mu \mathrm{m}$. Significance: ${ }^{*} P<0.05$, ${ }^{* *} P<0.01,{ }^{*}{ }^{*} P<0.001$ vs. the control group. ${ }^{*} P<0.05$ vs. the CAWS group

activation, which subsequently activated the NLRP3 inflammasome.

Cathepsin B, a member of the cathepsin protease family, resides mainly in lysosomes, and is released upon lysosomal rupture ${ }^{42}$. Lysosomal membranes can be permeabilized by a variety of means, including lysosomotropic detergents, viral proteins, toxin, ROS and so on ${ }^{43}$. Previous studies have reported that HMGB1 can permeabilize the phospholipid bilayer in the acidic environment of lysosomes, and trigger the leakage of LPS into the cyto$\mathrm{sol}^{44}$. Xu et al. also demonstrated that HMGB1 endocytosis leads to lysosome rupture, cathepsin B release, and subsequent caspase-1-mediated pyroptotic cell death in macrophages $^{24}$. Our data and previous studies have demonstrated that the serum level of HMGB1 is significantly increased in KD (Fig. S5b) ${ }^{26}$. Our results also showed that the level of HMGB1 in the supernatant medium and cultured THP1 cells was elevated (Fig. 5a and Fig. S5a). HMGB1 is known to be able to signal through the RAGE receptor, which are present in ECs. In $\mathrm{KD}$, it has been reported that RAGE mRNA expression is increased $^{26}$, and our data also showed that the mRNA and protein levels of RAGE in ECs were upregulated in KD conditions, which might be induced by elevated HMGB1 since HMGB1 internalization is RAGE-dependent, and RAGE deficiency can prevent HMGB1 endocytosis, and subsequent pyrotposis ${ }^{21}$. Furthermore, we found that either anti-HMGB1 antibody or RAGE inhibition in KD significantly ameliorated the inflammation in coronary arteries, decreased the expression of pyroptosisrelated proteins, and alleviated EC pyroptosis. Together, these results suggest that EC pyroptosis in KD is mediated by HMGB1/RAGE signaling, and is an important cause of coronary artery injury.

Collectively, our data provides new evidence that pyroptosis of ECs is a crucial player in the pathophysiology of KD. Our results demonstrate that HMGB1 released by immune cells triggers the HMGB1/RAGE signaling pathway in ECs, which then induces cathepsin B activation, subsequently activating canonical pyroptosis via the NLRP3 inflammasome. Given that KD is diagnosed using

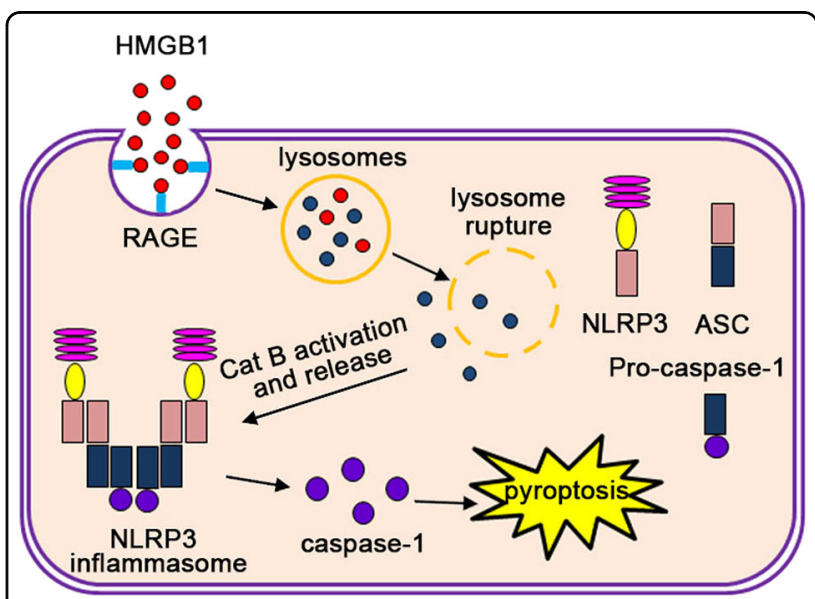

Fig. 8 Schematic model for endothelial cell pyroptosis in Kawasaki disease. High level of serum HMGB1 interacts with its receptor RAGE, enters lysosomes, and induces Cat B (cathepsin B) activation and release from the ruptured lysosomes. This is followed by NLRP3 inflammasome activation and subsequent induction of caspase-1-dependent pyroptosis

clinical criteria, and is thus prone to subjectivity, biomarkers that are specific to KD pathophysiology would be extremely valuable to optimize the management of $\mathrm{KD}$ patients. Our new findings suggest that circulating HMGB1 may be a sensitive predictor for EC damage in KD. Furthermore, mechanistic insights into KD outlined in this study also open new possibilities for therapeutic targets that act on inhibiting pyroptosis activation. These exciting new opportunities await further research and subsequent clinical translation. However, there are some limitations in our current study. Whether other factors or non-canonical pyroptosis signals are also involved in EC damage of KD remains uncertain, and requires further investigation. In addition, this study cannot exclude that other forms of cell death, such as apoptosis and necroptosis, also play an important role in pathophysiology of KD. Nevertheless, our current study presents novel evidence that firmly demonstrates the involvement of EC pyroptosis in KD. 


\section{Materials and methods}

\section{Patients' blood samples and ethical considerations}

Blood samples and clinical information were acquired from patients with KD and age-matched healthy controls at the Second Affiliated Hospital and Yuying Children's Hospital of Wenzhou Medical University between August 2017 and August 2019. The average age of KD patients and HCs was, respectively, 24.6 \pm 10.23 and $22.5 \pm$ 13.4 months. Healthy subjects were all children who received regular health checks and had no infections. Children that define KD met the criteria developed in 2017 by the American Heart Association ${ }^{3}$. All KD patients were scheduled to receive both aspirin $(30-50 \mathrm{mg} / \mathrm{kg} /$ day) and intravenous immunoglobin (IVIG, $2 \mathrm{~g} / \mathrm{kg}$ ). All these patients were IVIG-sensitive, and had no coronary aneurysm and additional comorbid medical conditions. Two microliters of serial blood specimens were collected from every KD patient in the acute phase (before IVIG therapy on days 3-7). All sera samples were stored at $-80^{\circ} \mathrm{C}$ within $4 \mathrm{~h}$ following collection until later use. All participants gave written consent for the use of their clinical information and blood samples for academic research. This research was approved by the ethics committee of Wenzhou Medical University, and conducted in accordance with the Helsinki Declaration.

\section{Enzyme-linked immunosorbent assay (ELISA)}

Serum concentrations of ASC, caspase-1, IL-1 $\beta$, IL-18, and GSDMD, and HMGB1 levels in the supernatant medium of THP1 cells were determined by the corresponding ELISA kits according to the manufacturer's instructions. ELISA kits for human ASC, human IL-1 $\beta$, human IL-18, and human GSDMD were acquired from ABclonal Biotechnology Co., Ltd. (Boston, MA, USA), and ELISA kits for human caspase-1 and human HMGB1 were purchased from Westang Bio-Technology Co., Ltd. (Shanghai, China). For examining HMGB1 levels in the THP1 cell supernatants, experiments were conducted as follows. Specifically, the $\mathrm{HC}$ and KD sera were removed and THP1 cells were washed with PBS after treatment with sera for $24 \mathrm{~h}$. Next, the THP1 cells continued to be incubated for the subsequent 24h. After that, HMGB1 levels were determined in the supernatants.

\section{Cell culture and treatments}

Human umbilical vein endothelial cells (HUVECs) and the human monocytic leukemia cell line, THP1, were purchased from American Type Culture Collection (ATCC, Manassas, VA, USA). Authentication of these cell lines was performed by the Genetic Testing Biotechnology Corporation (Suzhou, China) and the KeyCen BioTech (Nanjing, China) by short tandem repeat (STR) markers, and no mycoplasma contamination was detected. HUVECs were cultured in high glucose Dulbecco's modified Eagle's medium (DMEM) supplemented with $10 \%$ fetal bovine serum (FBS), and $1 \%(\mathrm{v} / \mathrm{v})$ pencillin/ streptomycin at $37{ }^{\circ} \mathrm{C}$ with $5 \% \mathrm{CO}_{2} / 95 \%$ air. HUVECs were plated in 6-, 24-well plates (Becton Dickinson Labware, Franklin Lakes, NJ) for different experiments, including LDH release, caspase-1 staining, TUNEL staining, cathepsin B staining and Hoechst 33342/PI staining. THP1 cells were maintained in $75-\mathrm{cm}^{2}$ plastic tissue culture flasks in RPMI-1640 medium with 10\% FBS. In the coculture system, HUVECs were cultured in the lower chamber, and the THP1 cells were placed in the upper chamber that permits diffusion of soluble molecules. Then $\mathrm{KD}$ or $\mathrm{HC}$ sera were added to the upper chamber and treated THP1 cells for $24 \mathrm{~h}$. At the same time, the HUVECs in the lower chamber would be affected by the diffusing molecules. All cultures were grown in DMEM medium. If necessary, the HUVECs would be pretreated with inhibitors for $30 \mathrm{~min}$, and then co-cultured with KD serum- or HC serum-treated THP1 cells for $24 \mathrm{~h}$.

\section{Western blot analysis}

Total proteins were extracted from heart tissues or endothelial cells using protein extraction reagents. Protein concentrations were quantified, and equal amounts of proteins $(60 \mu \mathrm{g})$ were separated by $12 \%$ SDS-PAGE gel and electro-transferred to nitrocellulose membranes. After blocking with $5 \%$ skimmed milk for $2 \mathrm{~h}$, membranes were incubated with the following primary antibodies at $4{ }^{\circ} \mathrm{C}$ overnight: NLRP3 (Proteintech, Chicago, USA, 1:1000, Cat. No.: 19771-1-AP), caspase-1 (Proteintech, Chicago, USA, 1:1000, Cat. No.: 22915-1-AP), GSDMD (Santa Cruz, USA, 1:500, Cat. No.: A2315), IL-1 $\beta$ (ABclonal, Boston, USA, 1:1000, Cat. No.: A1112), IL-18 (ABclonal, Boston, USA, 1:1000, Cat. No.: A1115), Transferrin (BIOSS, Beijing, China, 1:1000, Cat. No: bs2052R), or GAPDH (Proteintech, Chicago, USA, 1:2000, Cat. No: 60004-1-lg). After washing with TBST, membranes were incubated with HRP-conjugated secondary antibody $(1: 10,000)$ for $2 \mathrm{~h}$. Western blot bands were analyzed with the ChemiDicTM XRS + Imaging System (Bio-Rad Laboratories, Hercules, CA, USA), and the band densities were quantified with Multi Gauge Software of Science Lab 2006 (FUJIFILM Corporation, Tokyo, Japan).

\section{Immunofluorescence}

Immunofluorescence staining for endothelial cells was performed using standard protocols. In Brief, the cells were fixed with $4 \%$ paraformaldehyde for $30 \mathrm{~min}$, penetrated with $0.3 \%$ Triton X-100 for $1 \mathrm{~h}$, and then blocked with $5 \%$ bovine albumin for $30 \mathrm{~min}$. Subsequently, cells were incubated with anti-caspase- 1 antibody (Proteintech, Chicago, USA, 1:50, Cat. No.: 22915-1-AP) at $4{ }^{\circ} \mathrm{C}$ overnight, followed by incubation at $37^{\circ} \mathrm{C}$ with an Alexa Fluor 
594 donkey anti-rabbit secondary antibody (Abcam, USA, 1:1000, Cat. No.: ab150076) for $1 \mathrm{~h}$. Nuclei were stained by DAPI (Beyotime, China) for $20 \mathrm{~min}$. Then the cells were photographed under a laser scanning confocal microscope (Nikon, A1 PLUS, Tokyo, Japan).

\section{DNA fragmentation evaluation}

DNA fragmentation of endothelial cells was measured by TUNEL staining assay as previously described ${ }^{13}$. Briefly, endothelial cells were cultured on coverslips in a 24-well plate. After the indicated treatments, the cells were fixed with $4 \%$ paraformaldehyde and permeabilized with $0.3 \%$ Triton X-100. After washing with PBS, cells were incubated with TUNEL reaction mixture at $37^{\circ} \mathrm{C}$ in the dark for $1 \mathrm{~h}$, and stained by DAPI. Then cells were examined under a confocal laser scanning microscope (Nikon, A1 PLUS, Tokyo, Japan).

\section{Cell death assay}

Pyroptotic cell death in endothelial cells was assessed using LDH release assay and Hoechst 33342/PI staining ${ }^{11}$. After treatment with sera for $24 \mathrm{~h}$, cell culture medium was replaced with DMEM. After 6-h exposure, the supernatants from the treated cells were collected and centrifuged $(400 \times g, 5 \mathrm{~min})$. Then $120 \mu \mathrm{l}$ of the supernatant from each sample was transferred to a new 96-well plate and mixed with $60 \mu \mathrm{l}$ reaction mixture $(20 \mu \mathrm{l}$ lactate, $20 \mu \mathrm{l} \mathrm{INT}$, and $20 \mu \mathrm{l}$ diaphorase) for $30 \mathrm{~min}$ at room temperature. Serum-free medium was used as the $0 \%$ control and lysates of the untreated cell were used as the $100 \%$ maximal release. The absorbance was measured at $450 \mathrm{~nm}$ on a spectrophotometric microplate reader. For Hoechst 33342/PI staining (Beyotime Institute of Biotechnology, Haimen, China), the treated cells were collected and resuspended in staining buffer, then incubated with a mixed solution of Hoechst 33342 and PI for $25 \mathrm{~min}$ at $4{ }^{\circ} \mathrm{C}$. After that, the cells were photographed under an inverted fluorescence microscope (Nikon, TE-2000 U).

\section{Preparation of CAWS}

The CAWS were prepared from Candida albicans strain NBRC1385 by previously described methods ${ }^{5,40,45}$. Briefly, C. albicans cultures were incubated in C-limiting medium at $27^{\circ} \mathrm{C}$ for 2 days at a rotation speed of $270 \mathrm{rpm}$. After that, an equal volume of ethanol was added and put in a refrigerator at $4{ }^{\circ} \mathrm{C}$ overnight. Then the cultures were collected by centrifugation, and the pellet was dissolved in water with stirring for $2 \mathrm{~h}$. Next, the complex was centrifuged again, and the soluble fraction was harvested and mixed with an equal volume of ethanol, and allowed to stand undisturbed overnight. Finally, the complex was centrifuged, and the precipitate was acquired to dry with acetone. The obtained CAWS were dissolved in $0.9 \%$ normal saline and autoclaved before use.

\section{Animals and ethics statements}

For animal models of KD, male C57BL/6 mice (3-4 weeks of age) obtained from Wenzhou Medical University, License No. SCXK [ZJ] 2005-0019, were kept at a standard experiment cage in controlled conditions with temperature of $23 \pm$ $2{ }^{\circ} \mathrm{C}$, and humidity of $50 \pm 5 \%$. Mice were divided into six groups ( $n=6$ for each group) using a method of randomization: PBS group, CAWS group, CAWS + MCC950 group, CAWS + CA074-Me group, CAWS + FPS-ZM1 group, and CAWS + Anti-HMGB1 group. For groups designated for inhibitor pretreatment, inhibitors were injected $60 \mathrm{~min}$ before CAWS ( $4 \mathrm{mg} /$ body) were intraperitoneally administered. These procedures were repeated for 4 days. At day 28 post-final CAWS injection, the mice were anesthetized and killed for harvest of heart tissues and follow-up examinations. All experiments were conducted according to the Guide for the Care and Use of Laboratory Animals of the China National Institutes of Health, and were authorized by the Animal Care and Use Committee of Wenzhou Medical University (wydw 2017-0046).

\section{Histology and immunostaining}

Animal hearts were harvested and then fixed with 4\% paraformaldehyde and embedded with paraffin. $5 \mu \mathrm{m}$ thick sections were obtained, and HE staining was performed using standard protocols. TUNEL staining and immunostaining of caspase-1 and CD31 (endothelial cell marker) on the coronary arteries were also done. Mouse monoclonal anti-CD31 antibody (ab24950) and an Alexa Fluor 594 donkey anti-mouse secondary antibody (ab150108) were purchased from Abcam (USA). Images were examined under a laser scanning confocal microscope (Nikon, A1 PLUS, Tokyo, Japan).

\section{Data analysis}

Data were presented as mean $\pm \mathrm{SD}$, and were analyzed using SPSS version 17.0. All the data were normally distributed. A two-tailed unpaired Student's $t$-test was conducted to compare two experimental groups, and one way analysis of variance (ANOVA) followed by Duncan's multiple-range test was performed for the comparison of more than two groups. $P$-values less than 0.05 were considered as statistically significant. Sample size was chosen according to previous reports ${ }^{2,5,7}$, which performed similar experiments to observe significant results. Variance was similar between the groups that were being statistically compared.

\section{Acknowledgements}

We are grateful to Prof. Ian P. Wicks and Jacinta Hansen (Royal Melbourne Hospital \& University of Melbourne) for the methods of preparing CAWS. This research was supported by the Natural Science Foundation of China (No. 81970435, No. 81770502, and No. 81601705), Zhejiang Provincial

Natural Science Foundation of China (No. LQ18C010003, No. LY18H020012, and No. LQ15H020006), Zhejiang Provincial Science and Technology Project 
of Traditional Chinese Medicine (No. 2018ZZ019), and Wenzhou Science \& Technology Bureau Foundation (No. Y20190064, and No. Y20180007).

\begin{abstract}
Author details
${ }^{1}$ Pediatric Research Institute, The Second Affiliated Hospital and Yuying Children's Hospital of Wenzhou Medical University, Wenzhou 325027, China. ${ }^{2}$ Children's Heart Center, Institute of Cardiovascular Development and Translational Medicine, The Second Affiliated Hospital and Yuying Children's Hospital of Wenzhou Medical University, Wenzhou 325027, China. ${ }^{3}$ University of Maryland School of Medicine, Baltimore, MD 21201, USA. ${ }^{4}$ Department of Orthopaedics, The Second Affiliated Hospital and Yuying Children's Hospital of Wenzhou Medical University, Wenzhou 325027, China. ${ }^{5}$ Department of Pathophysiology, School of Basic Medicine Science, Wenzhou Medical University, Wenzhou, Zhejiang 325000, PR China. ${ }^{6}$ Pharmacology, School of Pharmacy, Wenzhou Medical University, Wenzhou, Zhejiang Province, China
\end{abstract}

\section{Conflict of interest}

The authors declare that they have no conflict of interest.

\section{Publisher's note}

Springer Nature remains neutral with regard to jurisdictional claims in published maps and institutional affiliations.

Supplementary Information accompanies this paper at (https://doi.org/ 10.1038/s41419-019-2021-3).

Received: 29 May 2019 Revised: 18 September 2019 Accepted: 25 September 2019

Published online: 14 October 2019

\section{References}

1. Uehara, R. \& Belay, E. D. Epidemiology of Kawasaki disease in Asia, Europe, and the United States. J. Epidemiol. 22, 79-85 (2012).

2. He, M. et al. miR-483 targeting of CTGF suppresses endothelial-tomesenchymal transition novelty and significance. Circulation Res. 120 354-365 (2017)

3. McCrindle, B. W. et al. Diagnosis, treatment, and long-term management of Kawasaki disease: a scientific statement for health professionals from the American Heart Association. Circulation 135, e927-e999 (2017).

4. Ueno, K. et al. Disruption of endothelial cell homeostasis plays a key role in the early pathogenesis of coronary artery abnormalities in Kawasaki disease. Sci. Rep. 7, 43719 (2017)

5. Stock, A. T., Hansen, J. A., Sleeman, M. A., McKenzie, B. S. \& Wicks, I. P. GM-CSF primes cardiac inflammation in a mouse model of Kawasaki disease. J. Exp. Med. 213, 1983-1998 (2016).

6. Wu, R. et al. miR-186, a serum microRNA, induces endothelial cell apoptosis by targeting SMAD6 in Kawasaki disease. Int. J. Mol. Med. 41 1899-1908 (2018).

7. Chu, M. et al. Bone marrow-derived microRNA-223 works as an endocrine genetic signal in vascular endothelial cells and participates in vascular injury from Kawasaki disease. J. Am. Heart Assoc. 6, e004878 (2017).

8. Furukawa, S. et al. Peripheral blood monocyte/macrophages and serum tumor necrosis factor in Kawasaki disease. Clin. Immunol. Immunopathol. 48 247-251 (1988)

9. Ichiyama, T. et al. NF-KB activation in peripheral blood monocytes/macrophages and T cells during acute Kawasaki disease. Clin. Immunol. 99, 373-377 (2001).

10. Chanput, W., Mes, J. J. \& Wichers, H. J. THP-1 cell line: an in vitro cell model for immune modulation approach. Int. Immunopharmacol. 23 37-45 (2014)

11. Leung, D. M. et al. Endothelial cell activation and high interleukin-1 secretion in the pathogenesis of acute Kawasaki disease. Lancet $\mathbf{3 3 4}$ 1298-1302 (1989)

12. Nakatani, K. et al. Circulating endothelial cells in Kawasaki disease. Clin. Exp. Immunol. 131, 536-540 (2003).

13. Jia, C. et al. Role of pyroptosis in cardiovascular diseases. Int. Immunopharmacol. 67, 311-318 (2019).
14. Wu, X. et al. Nicotine promotes atherosclerosis via ROS-NLRP3-mediated endothelial cell pyroptosis. Cell Death Dis. 9, 171 (2018).

15. Zhang, Y. et al. Melatonin prevents endothelial cell pyroptosis via regulation of long noncoding RNA MEG3/miR-223/NLRP3 axis. J. Pineal Res. 64, e12449 (2018).

16. Li, X. et al. MicroRNA-30d regulates cardiomyocyte pyroptosis by directly targeting foxo3a in diabetic cardiomyopathy. Cell Death Dis. 5, e1479 (2014).

17. Qiu, Z. et al. NLRP3 inflammasome activation-mediated pyroptosis aggravates myocardial ischemia/reperfusion injury in diabetic rats. Oxid. Med. Cell Longev. 2017, 9743280 (2017).

18. Chen, $Y$. et al. Endothelial Nlrp3 inflammasome activation associated with lysosomal destabilization during coronary arteritis. BBA - Mol. Cell Res. 1853 396-408 (2015)

19. Shi, J., Gao, W. \& Shao, F. Pyroptosis: gasdermin-mediated programmed necrotic cell death. Trends Biochemical Sci. 42, 245-254 (2017).

20. Yu, Y., Tang, D. \& Kang, R. Oxidative stress-mediated HMGB1 biology. Front. Physiol. 6, 93 (2015)

21. Luan, Z. G. et al. HMGB1 activates nuclear factor-KB signaling by RAGE and increases the production of TNF-a in human umbilical vein endothelial cells. Immunobiology 215, 956-962 (2010).

22. Geng, Y. et al. Heatstroke induces liver injury via IL-1 $\beta$ and HMGB1-induced pyroptosis. J. Hepatol. 63, 622-633 (2015)

23. Tang, D. et al. HMGB1 release and redox regulates autophagy and apoptosis in cancer cells. Oncogene 29, 5299 (2010).

24. $\mathrm{Xu}$, J. et al. Macrophage endocytosis of high-mobility group box 1 triggers pyroptosis. Cell Death Differ. 21, 1229 (2014).

25. Tian, J. et al. Toll-like receptor 9-dependent activation by DNA-containing immune complexes is mediated by HMGB1 and RAGE. Nat. Immunol. 8, 487 (2007).

26. Hoshina, T. et al. High mobility group box 1 (HMGB1) and macrophage migration inhibitory factor (MIF) in Kawasaki disease. Scand. J. Rheumatol. 37, 445-449 (2008).

27. Xu, Y.-J., Zheng, L., Hu, Y.-W. \& Wang, Q. Pyroptosis and its relationship to atherosclerosis. Clin. Chim. Acta 476, 28-37 (2018).

28. Gross, 0 . et al. Syk kinase signalling couples to the Nlrp3 inflammasome for anti-fungal host defence. Nature 459, 433 (2009).

29. Bruchard, $M$. et al. Chemotherapy-triggered cathepsin B release in myeloidderived suppressor cells activates the Nlrp3 inflammasome and promotes tumor growth. Nat. Med. 19, 57-64 (2013).

30. Liu, Z. et al. Melatonin alleviates inflammasome-induced pyroptosis through inhibiting NF-kB/GSDMD signal in mice adipose tissue. J. Pineal Res. 63, e12414 (2017).

31. Ichiyama, T. et al. An immunoglobulin agent (IVIG) inhibits NF-kB activation in cultured endothelial cells of coronary arteries in vitro. Inflamm. Res. $\mathbf{5 3}$ 253-256 (2004).

32. Fink, S. L., Bergsbaken, T. \& Cookson, B. T. Anthrax lethal toxin and Salmonella elicit the common cell death pathway of caspase-1dependent pyroptosis via distinct mechanisms. Proc. Natl Acad. Sci. USA 105, 4312-4317 (2008)

33. Lev, M. et al. Microbiota-modulated metabolites shape the intestinal microenvironment by regulating NLRP6 inflammasome signaling. Cell 163 1428-1443 (2015).

34. Tenthorey, J. L., Kofoed, E. M., Daugherty, M. D., Malik, H. S. \& Vance, R. E. Molecular basis for specific recognition of bacterial ligands by NAIP/NLRC4 inflammasomes. Mol. Cell 54, 17-29 (2014).

35. Zhu, S. et al. Nlrp9b inflammasome restricts rotavirus infection in intestinal epithelial cells. Nature 546, 667 (2017).

36. Hornung, $\mathrm{V}$. et al. AIM2 recognizes cytosolic dsDNA and forms a caspase-1activating inflammasome with ASC. Nature 458, 514 (2009).

37. Heilig, R. \& Broz, P. Function and mechanism of the pyrin inflammasome. Eur. J. Immunol. 48, 230-238 (2018)

38. Pétrilli, V., Dostert, C., Muruve, D. A. \& Tschopp, J. The inflammasome: a danger sensing complex triggering innate immunity. Curr. Opin. Immunol. 19, 615-622 (2007)

39. Duncan, J. A. et al. Neisseria gonorrhoeae activates the proteinase cathepsin B to mediate the signaling activities of the NLRP3 and ASC-containing inflammasome. J. Immunol. 182, 6460-6469 (2009).

40. Zhang, Q. et al. Cathepsin B Regulates the Activation of NLRP3 Inflammasome by Calcium Signaling. Neuropsychiatry (Lond.) 8, 1899-1912 (2018). 
41. Bai, $H$. et al. Cathepsin B links oxidative stress to the activation of NLRP3 inflammasome. Exp. Cell Res. 362, 180-187 (2018).

42. Turk, V., Turk, B. \& Turk, D. Lysosomal cysteine proteases: facts and opportunities. EMBO J. 20, 4629-4633 (2001).

43. Aits, S. \& Jäättelä, M. Lysosomal Cell Death at a Glance. (The Company of Biologists Ltd, 2013).
44. Deng, M. et al. The endotoxin delivery protein HMGB1 mediates caspase-11dependent lethality in sepsis. Immunity 49, 740-753. e747 (2018).

45. Tada, R., Nagi-Miura, N., Adachi, Y. \& Ohno, N. The influence of culture conditions on vasculitis and anaphylactoid shock induced by fungal pathogen Candida albicans cell wall extract in mice. Microb. Pathogenesis 44, 379-388 (2008). 